\title{
Characteristics of wheel-rail vibration of the vertical section in high-speed railways
}

\author{
Jiuchuan YANG ${ }^{1}$, Kaiyun WANG ${ }^{1 *}$, Hongyu CHEN $^{2}$ \\ 1. Traction Power State Key Laboratory, Southwest Jiaotong University, Chengdu 610031, China \\ 2. School of Mechanical Engineering, Southwest Jiaotong University, Chengdu 610031, China
}

\begin{abstract}
In order to analyze the characteristics of wheel-rail vibration of the vertical section in a high-speed railway, a vehicle-line dynamics model is established using the dynamics software SIMPACK. Through this model, the paper analyzes the influence of vertical section parameters, including vertical section slope and vertical curve radius, on wheel-rail dynamics interaction and the acting region of wheel-rail vibration. In addition, the characteristics of wheelrail vibration of the vertical section under different velocities are investigated. The results show that the variation of wheel load is not sensitive to the vertical section slope but is greatly affected by the vertical curve radius. It was also observed that the smaller the vertical curve radius is, the more severe the interaction between the wheel and rail becomes. Furthermore, the acting region of wheel-rail vibration expands with the vertical curve radius increasing. On another note, it is necessary to match the slope and vertical curve radius reasonably, on account of the influence of operation speed on the characteristics of wheel-rail vibration. This is especially important at the design stage of vertical sections for lines of different grades.
\end{abstract}

Key words: high-speed railway; vertical section; wheel-rail vibration; slope; vertical curve radius

(C) 2011 JMT. All rights reserved.

\section{Introduction}

$\mathrm{W}$ ith the rapid development of high-speed railways, extensive research into railway technologies is warranted, especially on the aspect of dynamic interactions between vehicle and railway line, to guarantee the operational security of vehicles at high speed and improve the passenger comfort. The increase in the severity of the dynamic interaction between wheel and rail stems from the increasing speed of trains. As a result, it is necessary to analyze the characteristics of wheel-rail vibration. The vertical curve is an important component of a railway line and is usually the very source of wheelrail vibration, especially at the time when a vehicle rapidly passes through a railway line, and will seriously affect the safety and comfort of the vehicle. At present, the usual practice is to only take some limit values to

This paper was presented at the 5th International Symposium on Environmental Vibration in Chengdu, China, October 2022,2011 , and recommended by the scientific committee of the symposium to JMT

Received Jan. 15, 2012; revision accepted Feb. 27, 2012

${ }^{*}$ Corresponding author. Tel.:+86-28-87600773

E-mail: kywang@home.swjtu.edu.cn (K.Y.WANG)

(C) 2011 JMT. All rights reserved

doi: 10.3969/j.issn.2095-087X.2012.01.002 design the vertical section of railway lines, such as the maximum slope and the minimum slope length, according to the grade of a certain line [1-3]. Moreover, the existing studies on the vertical curve focused more on the vertical section parameters without considering the influence of these parameters on the dynamic interaction between the vehicle and line [4-6]. The aforementioned studies involve either static or quasi-static analysis. There is little literature available on using a systematic method based on dynamics to study wheel-rail vibration, and some related issues are mainly the assessment making for a certain line [7-9]. Considering the research conducted in China addressing this issue, most researchers fail to consider the vertical centrifugal acceleration of the high-speed vehicle, and the permissible maximum value can only be found in the latest European norm ENV13801-1 [10].

Based on the multi-body dynamics and the existing achievements [11-13], this paper aims to systematically investigate the influence of vertical section parameters on the characteristics of wheel-rail vibration and discuss the relationship between the acting region of wheel-rail vibration and these parameters. Furthermore, the characteristics of wheel-rail vibration at different velocities are investigated. All the work carried out may contribute to the design of the vertical section of high-speed railways [14]. 


\section{Vertical section}

The railway line could be regarded as the trajectory of a track center moving along a spatial curve. Thus, it can be described by a horizontal section and a vertical section. The vertical section describes the upright variation of the line, which contains the following components, i.e. a straight line, a vertical curve, and the dummy point of slope change (Fig. 1). The vertical curve is used to connect two adjacent straight lines whose slope changes more than $1 \%$ and contributes to mitigating the vibration between the vehicle and line.

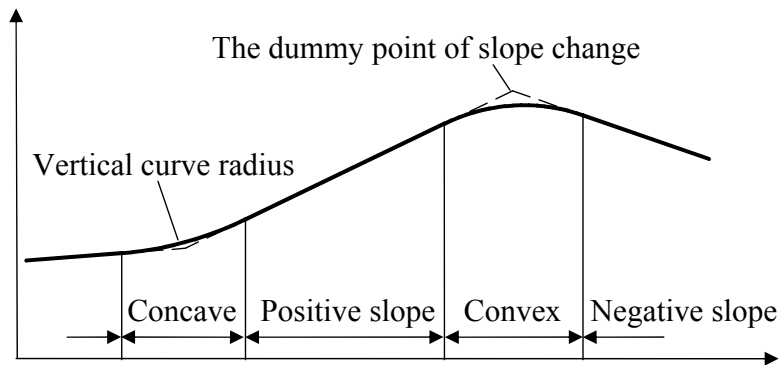

Fig. 1 Components of vertical section

Vertical section parameters include slope and vertical curve radius. According to the upright variation of line, the slope could be classified into positive and negative ones, while the vertical curve into concave and convex ones.

\section{Dynamics model}

A vehicle-line dynamics model (Fig. 2) is established using the software SIMPACK which is based on multibody dynamics. The parameters of the vehicle model are based on a certain type of Chinese railway high-speed vehicle. Concave and convex vertical curve models are established as shown in Fig. 3, and are studied on the characteristics of wheel-rail vibration. In the two different conditions, the major discussions of the vertical dynamic performances are mainly on the aspects of wheel-rail vertical force and vertical centrifugal acceleration of the wheel set. To ensure the clarity of results, there is no track irregularity applied in the simulation.

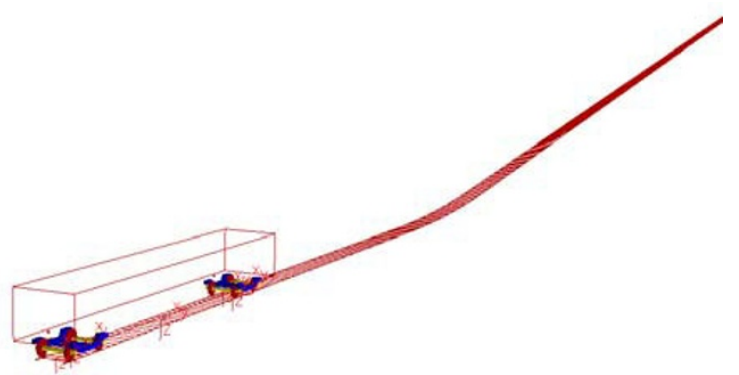

Fig. 2 Vehicle-line dynamics model

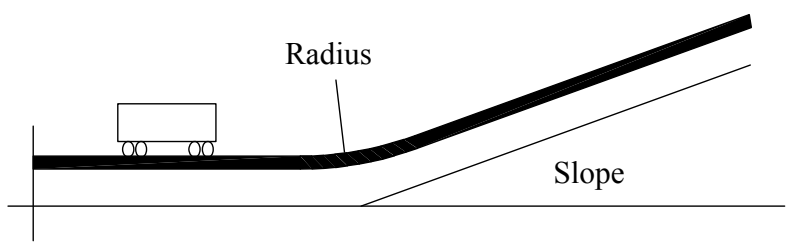

(a) Concave vertical curve model

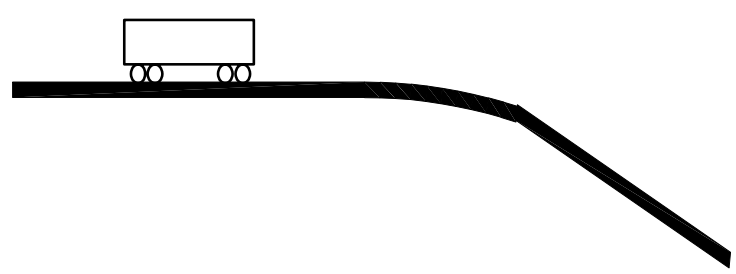

(b) Convex vertical curve model

Fig. 3 Vertical curve models

\section{Wheel-rail vibration of vertical section}

\subsection{Single influence of vertical section parameters}

We analyzed the characteristics of wheel-rail vibration under the effect of single parameters. Shown in the list of Table 1 to Table 4 are the calculation conditions with the variation of either slope or vertical curve radius.

Table 1 Calculation conditions of changing concave vertical curve radius

\begin{tabular}{lrrrrr}
\hline Calculation condition & $1-1$ & $1-2$ & $1-3$ & $1-4$ & $1-5$ \\
\hline Slope (\%) & & & 5 & & \\
Radius (km) & 10 & 15 & 20 & 25 & 30 \\
\hline
\end{tabular}

Table 2 Calculation conditions of changing convex vertical curve radius

\begin{tabular}{lccccc}
\hline Calculation condition & $2-1$ & $2-2$ & $2-3$ & $2-4$ & $2-5$ \\
\hline Slope (\%) & & & -5 & & \\
Radius (km) & 10 & 15 & 20 & 25 & 30 \\
\hline
\end{tabular}

Table 3 Calculation conditions of changing positive slope

\begin{tabular}{lcccc}
\hline Calculation condition & $3-1$ & $3-2$ & $3-3$ & $3-4$ \\
\hline Slope (\%) & 5 & 10 & 15 & 20 \\
Radius (km) & \multicolumn{5}{c}{30} \\
\hline
\end{tabular}

Table 4 Calculation conditions of changing negative slope

\begin{tabular}{lcccc}
\hline Calculation condition & $3-1$ & $3-2$ & $3-3$ & $3-4$ \\
\hline Slope (\%) & -5 & -10 & -15 & -20 \\
Radius (km) & \multicolumn{3}{c}{30} \\
\hline
\end{tabular}


Fig. 4 shows the wheel-rail vertical force changing with the radius as the vehicle passes through different concave vertical curves (Table 1) at a speed of $300 \mathrm{~km} / \mathrm{h}$. A phenomenon of wheel load increments is presented clearly, and the increment of the wheel load is larger when the radius is smaller. For example, the increment of the wheel load is $2.7 \mathrm{kN}$ when the radius is $10 \mathrm{~km}$, but it is only $0.8 \mathrm{kN}$ when the radius increases to $30 \mathrm{~km}$.

Fig. 5 shows the calculation results of the vehicle passing through different convex vertical curves (Table 2) at the same speed of $300 \mathrm{~km} / \mathrm{h}$. The results show that the wheel load appears to decrease under these conditions. The decrease of the wheel load becomes gradually larger with the reduction of the radius. For example, when the radius is $10 \mathrm{~km}$, the decrease in the wheel load is as high as $5 \mathrm{kN}$; but it falls to $1.5 \mathrm{kN}$ when the radius approaches $30 \mathrm{~km}$.

Under the different slope conditions (Tables 3 and 4), the responses of wheel-rail vertical force in a time domain are shown in Fig. 6 and Fig. 7. It is obvious that the phenomenon of the wheel load increase and decrease also appears. In Fig. 6, the values of all the increments

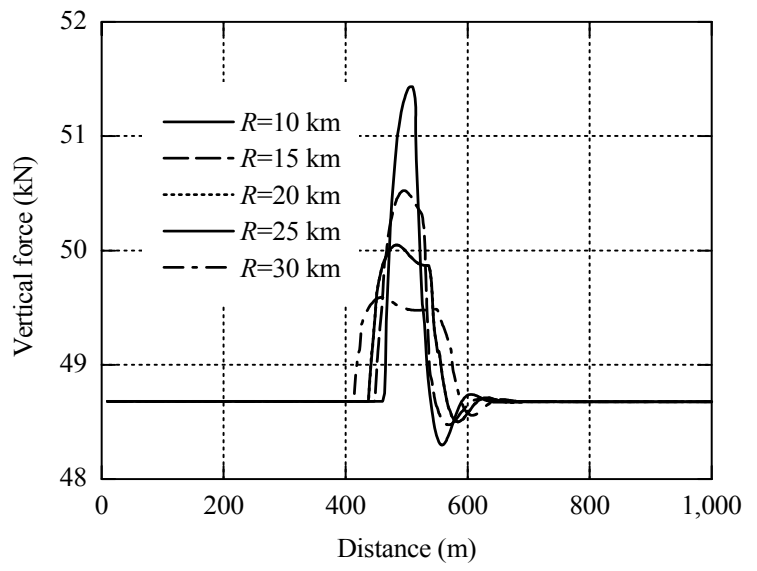

Fig. 4 Influence of concave vertical curve radius

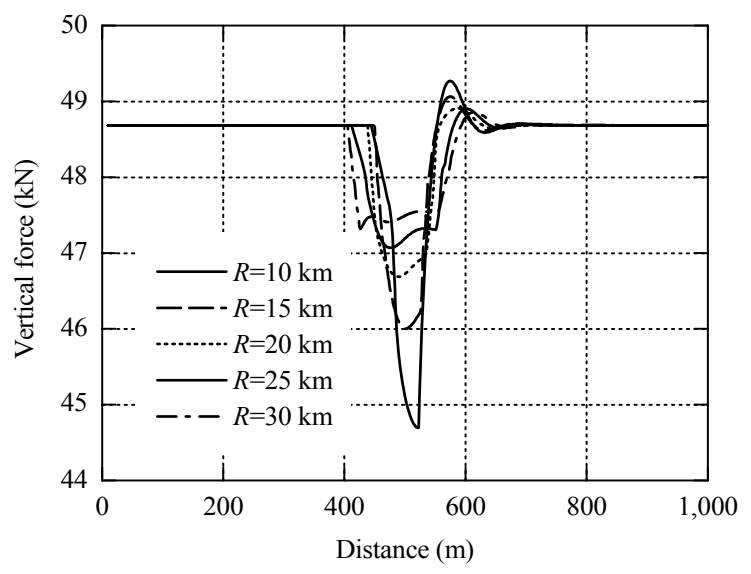

Fig. 5 Influence of convex vertical curve radius

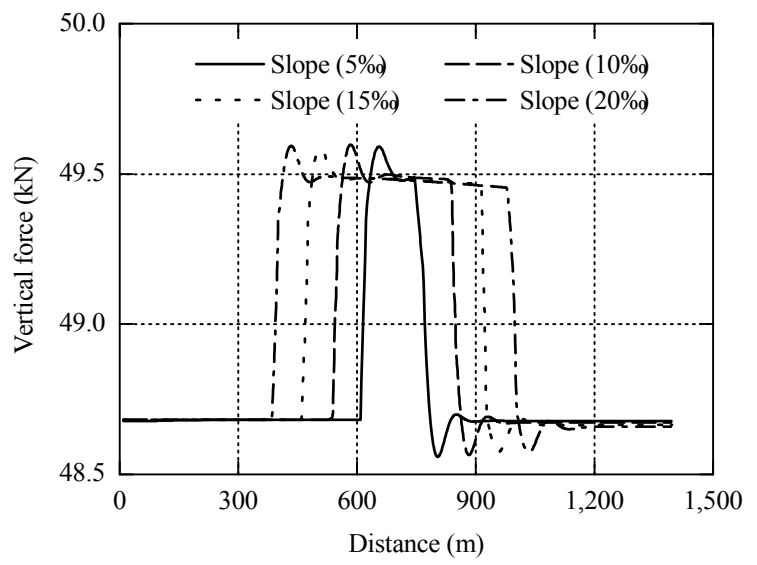

Fig. 6 Influence of positive slope

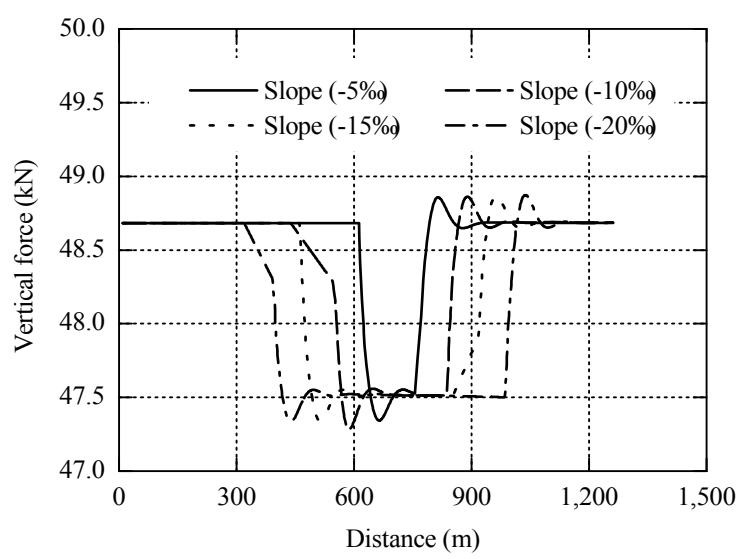

Fig. 7 Influence of negative slope

are $0.8 \mathrm{kN}$ irrespective of the value of the positive slope. In a similar fashion, Fig. 7 shows that the magnitude of decrease, in all negative slope conditions, is almost the same value of $1.5 \mathrm{kN}$. Certainly, the line environment, traction and braking ability, construction cost, and other factors should be taken into account in designing the largest slope [15]. Besides, it is noticeable that the wheel-rail vibration lasts longer when the slope increases.

According to the analysis mentioned above, we can see that the variation of wheel load is not sensitive to slope but is seriously affected by the vertical curve radius.

\subsection{Complex influence of vertical section parameters}

In order to understand the characteristics of wheelrail vibration further, just taking the concave vertical curve as an example, we designed various calculation conditions of the vertical section by combining different slopes and vertical curve radii.

Fig. 8 shows the maximum increment of wheel load in different conditions when the vehicle passes through the vertical curves at the speed of $300 \mathrm{~km} / \mathrm{h}$. There is 
almost no change in wheel load with the increase of the slope from $5 \%$ o to $20 \%$; however, the maximum increment decreases non-linearly as the vertical curve radius increases. For instance, when the vertical curve radius increases from 5 to $10 \mathrm{~km}$, the rate of change can reach up to $51 \%$; when the radius increases from 20 to $25 \mathrm{~km}$, the rate falls to $20 \%$; and it is only $13 \%$ when the radius increases from 35 to $40 \mathrm{~km}$. It is obvious that the wheel load increases sharply when the vertical curve radius is smaller than $15 \mathrm{~km}$.

To ensure operational safety and comfort, the vertical centrifugal acceleration of the wheel set should not be large. The maximum values of vertical centrifugal acceleration of the wheel set are shown in Fig. 9. It varies similarly to the increment of wheel load (shown in Fig. 8). When the vertical curve radius is smaller than $15 \mathrm{~km}$, the wheel set has a very large vertical centrifugal acceleration. For example, when the radius is $5 \mathrm{~km}$, the maximum value is close to $1 \mathrm{~m} / \mathrm{s}^{2}$; and when the radius is $10 \mathrm{~km}$, it approaches $0.5 \mathrm{~m} / \mathrm{s}^{2}$. It is noticeable that those values are beyond the permitted value of $0.35 \mathrm{~m} / \mathrm{s}^{2}$ in European norm ENV13801-1. Therefore, for a line with a maximum speed of $300 \mathrm{~km} / \mathrm{h}$, the minimum value of the vertical curve radius should be no less than $15 \mathrm{~km}$.

\section{Research of wheel-rail vibration acting region}

As Fig. 6 and Fig. 7 show, the acting region of wheelrail vertical force expands as the slope increases. If the wheel-rail force lasts longer, it will weaken the safety and comfort of vehicles. Therefore, we define a physical variable $T$ in units of $\mathrm{m}$ to describe the region of wheelrail vibration action, which is between the beginning and end of the wheel load variation. Then, the same calculation conditions as in Section 4.2 were applied.

Fig. 10 shows the relationship of the $T$ value with the slope and vertical curve radius. It can be seen that the $T$ value increases linearly either with the slope or the vertical curve radius increasing.

According to the calculation results, when the slope is $5 \%$ and the vertical curve radius is $20 \mathrm{~km}, T$ is $130 \mathrm{~m}$; when the values are $15 \%$ and $25 \mathrm{~km}$, respectively, $T$ is $395 \mathrm{~m}$. It can be concluded that the acting region of wheel-rail vibration is approximately equal to the product of slope and vertical curve radius, shown as Eq. (1):

$$
T \approx i \cdot R,
$$

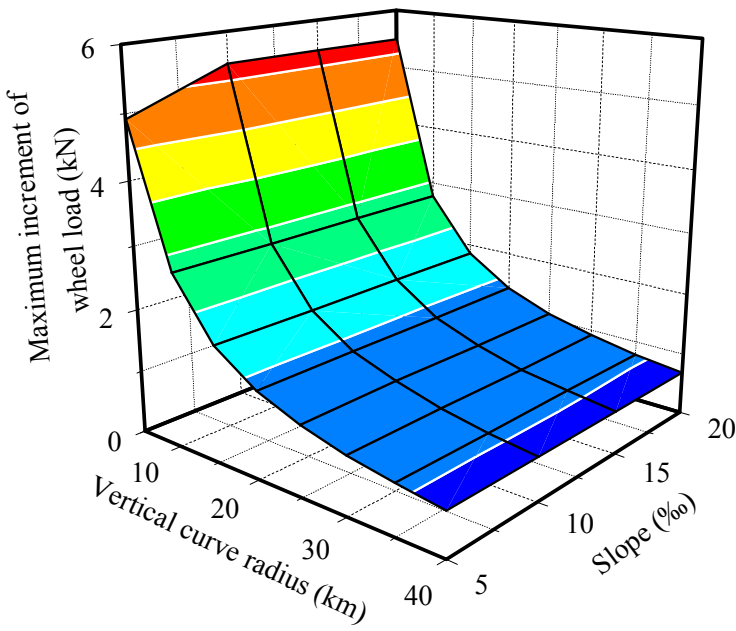

Fig. 8 Maximum increment of wheel load

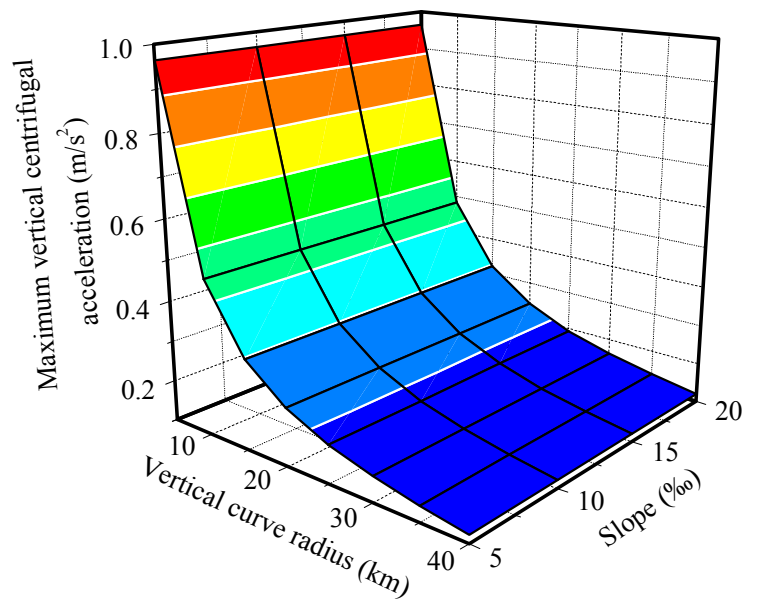

Fig. 9 Maximum vertical centrifugal acceleration of wheel set

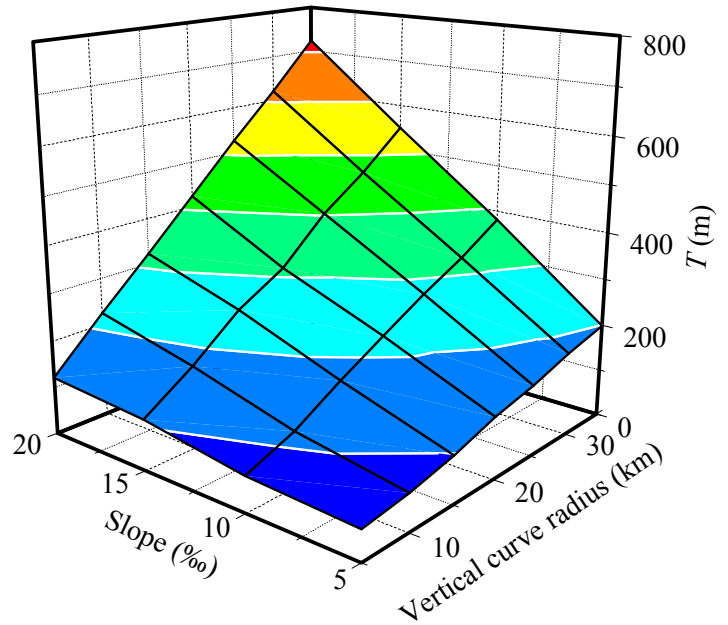

Fig. 10 Relationship of $T$ value with the slope and vertical curve radius 


\section{Influence of velocity on the wheel-rail vi- bration}

In order to investigate the influence of velocity on the characteristics of wheel-rail vibration, taking a certain vertical section as an example, we set the slope and the vertical curve radius as $5 \%$ and $20 \mathrm{~km}$, respectively; and conducted simulations at four different speeds of $200,250,300$, and $350 \mathrm{~km} / \mathrm{h}$.

Fig. 11 shows the maximum values of wheel-rail vertical force at different velocities. When the operation speed increases in order from 200 to $350 \mathrm{~km} / \mathrm{h}$, the maximum values increase linearly from 49.5 to $51.4 \mathrm{kN}$.

Fig. 12 shows the maximum values of vertical centrifugal acceleration of wheel set at different velocities. When the vehicle velocity is more than $300 \mathrm{~km} / \mathrm{h}$, the maximum values are all beyond the permitted value of $0.35 \mathrm{~m} / \mathrm{s}^{2}$. Therefore, according to the European norm, the vertical section parameters above are not suitable for the line with a maximum velocity of $350 \mathrm{~km} / \mathrm{h}$.

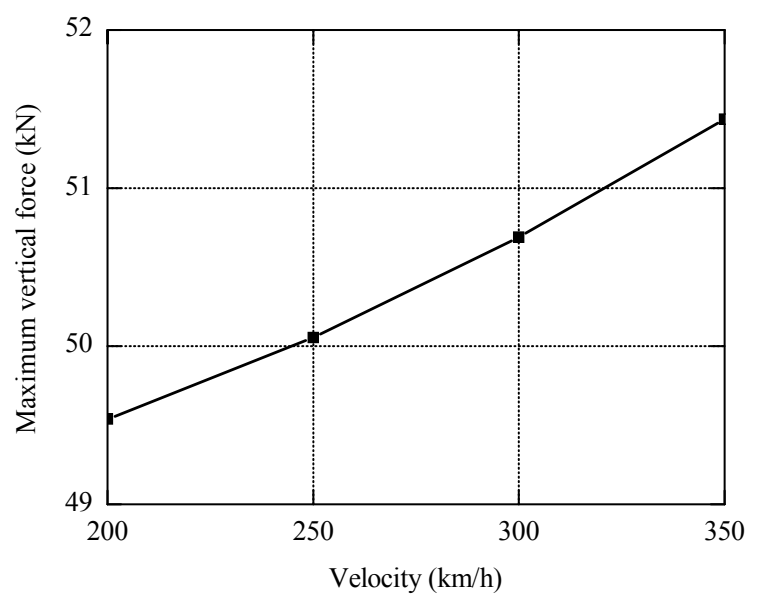

Fig. 11 Maximum wheel-rail vertical force at different velocities

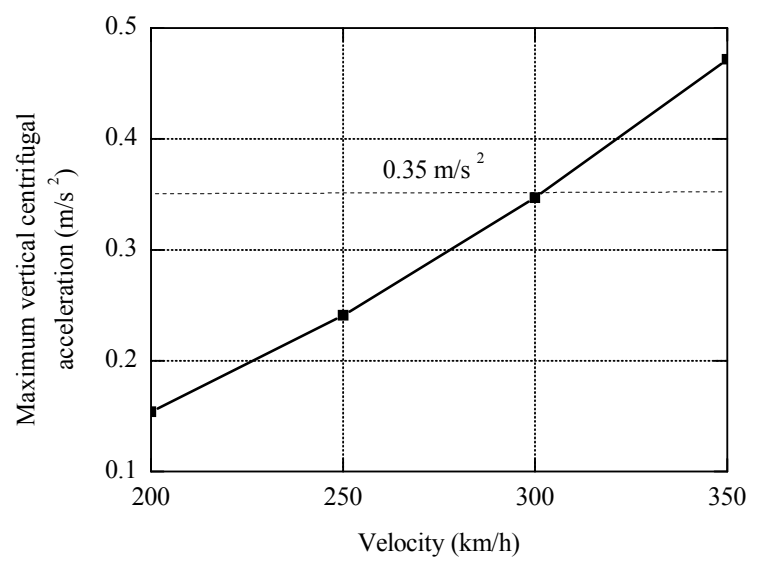

Fig. 12 Maximum vertical centrifugal acceleration of wheel set at different velocities
On a final note, at the stage of vertical section design for railway lines of different speeds, it is necessary to simulate the matching relationship between the slope and vertical curve radius, so as to determine the most reasonable condition.

\section{Conclusions}

(1) When a vehicle rapidly passes through the vertical curve, the wheel-rail vibration becomes severe. The value of vertical dynamics indexes, especially the variation of wheel load and the vertical centrifugal acceleration of the wheel set, are larger when the radius is smaller.

(2) For a line with a maximum speed of $300 \mathrm{~km} / \mathrm{h}$, the minimum value of vertical curve radius should be no less than $15 \mathrm{~km}$.

(3) Changing the slope has almost no influence on wheel-rail vertical dynamics performance, but the acting region of wheel-rail vibration expands with the increase of slope. The $T$ value is approximately equal to the product of slope and vertical curve radius.

(4) For the principles of designing vertical sections, it is recommended that when the slope is small, the vertical curve radius could be large, but when the slope is large, the radius could be decreased properly.

(5) As the main wheel-rail vertical dynamics indexes, the wheel-rail vertical force and the vertical centrifugal acceleration increase linearly with the increase of speed. Therefore, for railway lines of different grades, a detailed study on the matching relationships of slope and vertical curve radius based on wheel-rail dynamics is imperative.

\section{Acknowledgement}

The authors wish to acknowledge the support and motivation provided by the National Natural Science Foundation of China (No. 51075340), the Fok YingTong Education Foundation for Young Teachers in the Higher Education Institutions of China (No. 121075), and the Program for Innovation Research Team in University in China (No. IRT1178).

\section{References}

[1] B. Cheng, Research of maximum gradient value of Datong-Xi'an passenger dedicated railway. Shanxi Architecture, 2010, 36(4): 289-290 (in Chinese).

[2] W.K. Chen, Research on maximum gradient value of Zhengzhou-Wuhan passenger dedicated railway line, Railway Investigation and Surveying, 2008(1): 44-46 (in Chinese). 
[3] Y.J. Feng, J.R. Huang, Z.Q. Sheng, et al., The length for the minimum longitudinal gradient of railway, China Railway Science, 1995, 16(2): 81-91 (in Chinese).

[4] W. Bian, Discussion of Setting Vertical curve in the design of vertical section, Railway Science of Shanghai, 2003(3): 16-18.

[5] C.J. Jia, On exploration of design for speed-raising $200 \mathrm{~km} / \mathrm{h}$ plain vertical section of existing line, Shanxi Architecture, 2010, 36(26): 270-271 (in Chinese).

[6] H.W. He, Z.J. Liu, X.M. Diao, et al., Study and validation of the technical standard for the plane and profile sections of existing lines with $200 \mathrm{~km} / \mathrm{h}$ speed-raising, Journal of the China Railway Society, 2007, 29(2): 6470 (in Chinese).

[7] Z.H. Zhou, J. Xiang, X.Y. Liu and J. Zeng, Dynamics assessment of plane and profile section design parameters of Guangzhou-Zhuhai Intercity Railway, Journal of Railway Science and Engineering, 2009, 6(5): 21-25 (in Chinese).

[8] Routing Department of the Second Railway Design Institute, Full and comprehensive dynamic performance simulation assessment research reports of SuiningChongqing railway ballastless track test section, Chengdu, 2005 (in Chinese).

[9] China Academy of Railway Science, Design optimization and simulation analysis of plane and profile sections of Beijing-Shanghai high-speed railway, Beijing, 2005 (in Chinese).

[10] ENV 13801-1, Railway applications-track alignment design parameters-track gauges $1435 \mathrm{~mm}$ and wider-Part 1: Plain line, Brussels: European Committee for Standardization, 2002.

[11] K.Y. Wang, J.X. Liu, W.M. Zhai, et al., Running safety and comfortability simulation of railway, Journal of Traffic and Transportation Engineering, 2006, 6(3): 9-13(in Chinese).

[12] K.Y. Wang, W.J. Zhou, W.M. Zhai, et al., Study on the reasonable match of horizontal and longitudinal section of mixed railway above $200 \mathrm{~km} / \mathrm{h}$ based on dynamic theory, Railway Standard Design, 2005, 25(7): 1-3 (in Chinese)

[13] K.Y. Wang, W.J. Zhou, Comparison on dynamics performance of different sections of mixed railway with passenger and freight traffic, Journal of Railway Engineering Society, 2005(5): 1-4 (in Chinese).

[14] Y.Z. Wang, Some Discussion about Location Design Technology of High-Speed Railway [Dissertation], Chengdu: Southwest Jiaotong University, 2003 (in Chinese)

[15] Y. Zhu, Innovation and practice on railway location concept, Journal of Railway Engineering Society, 2009(6): 1-5 (in Chinese).

(Editor: Junsi LAN) 